\title{
Double-Active-Layer Index-Guided InGaAsP-InP Laser Diode
}

\author{
MARKUS KAJANTO, T. R. CHEN, Y. H. ZHUANG, AND AMNON YARIV, FEllow, IEEE
}

\begin{abstract}
A buried crescent InGaAsP-InP laser with two active layers was fabricated to study the temperature behavior of the doublecarrier-confinement structure. An anomalously high-characteristic temperature $T_{0}$ was measured and an optical switching behavior was observed. A model analysis and numerical calculation using a rate equation approach explained qualitatively very well the experimental results. It was revealed that not only the Auger recombination in this special double-active-layer configuration, but that the temperature dependent leakage current, which leads to uniform carrier distribution in both active regions, is essential to increase $T_{0}$.
\end{abstract}

\section{INTRODUCTION}

$T^{\prime}$ HERE has been continuous interest in making InGaAsP-InP lasers with high-characteristic temperature $T_{0}[1]-[5]$. The suggested approaches included highly doping the p-InP cladding layer [1]-[2]; using a quasitwo-dimensional active-layer structure [3]; and using a double-carrier-confinement (DCC) structure [4]-[5]. In the last structure a second active layer is grown to collect the carriers which leak out of the first active layer over the heterobarrier and to reuse them for radiative recombination. $T_{0}$ values of over $180 \mathrm{~K}$ have been reported [4][5]. However, the idea has only been demonstrated in broad-area (or wide-stripe) lasers so far. It is thus of considerable interest to see if the idea is applicable to the commonly used laser structures, e.g., buried heterostructure $(\mathrm{BH})$ or buried crescent $(\mathrm{BC})$ lasers.

In this paper, we report on experimental results from a buried-crescent double-carrier-confinement (BC-DCC) laser. In addition to the increase of characteristic temperature $T_{0}$, we found highly nonlinear behavior in the light versus current $(L-I)$ characteristics. A theoretical analysis explained the basic features of the experimental observations.

\section{Structure AND Fabrication}

A buried crescent structure was used in our study. In this structure, the injected carriers as well as the optical mode are well confined in two dimensions, which greatly

\footnotetext{
Manuscript received March 20,1990; revised June 19, 1990. This work was supported by the Defense Advanced Research Projects Agency and the Office of Naval Research.

M. Kajanto was with the Department of Applied Physics, California Institute of Technology, Pasadena, CA 91125 . He is now with McKinsey \& Company, Inc., S-10724, Stockholm, Sweden.

T. R. Chen, Y. H. Zhuang, and A. Yariv are with the Department of Applied Physics, California Institute of Technology, Pasadena, CA 91125.
}

IEEE Log Number 9039202. facilitates the theoretical analysis and quantitative calculations. Lasers were made both on $\mathrm{p}-\mathrm{InP}$ and $\mathrm{n}$-InP substrates. A schematic structure of the laser is shown in Fig. 1(a) and (b). A scanning electron microscope (SEM) photograph of the double active layers is shown in Fig. 1(c). The fabrication processes of the laser involve two-step liquid-phase epitaxy (LPE) growth which has been described in great detail in the literature [6]. The only difference is the growth of two active layers by the "threemelt technique" [5]. In the case of an n-InP substrate, during the second growth cycle, the n-InP lower cladding layer was grown first, followed by an undoped active layer and a $\mathrm{p}$-InP separation layer. The wafer was then pushed back across the InGaAsP solution for a second active-layer growth. The regrowth cycle was completed by growing a $\mathrm{p}$-InP cladding layer and a $\mathrm{p}^{+}-\mathrm{InGaAsP}$ cap layer. For a $\mathrm{BC}$-DCC laser on a $\mathrm{p}$-InP substrate, a similar technique was used during the regrowth. In the whole second growth process, only one InGaAsP melt is used for the growth of two active layers which ensures the exact overlap of the transition gain profile of the two layers. The thickness of the active layers is $0.15-0.25 \mu \mathrm{m}$, the separation between the two active layers is $0.1-0.3 \mu \mathrm{m}$ and the width of the two active layers is $2-2.5 \mu \mathrm{m}$.

\section{Performances of the BC-DCC Laser}

The threshold currents of the BC-DCC laser on an nInP substrate are in the range of $25-45 \mathrm{~mA}$ (for a cavity length of $\sim 250 \mu \mathrm{m}$ ). Lasers made on the same wafer with a single active layer had a threshold current of 10-15 mA. For BC-DCC laser on $\mathrm{p}$-InP substrate, the threshold currents are $40-60 \mathrm{~mA}$ while lasers made from the same solution with single active layer had $I_{\mathrm{th}}$ of typically 15-20 $\mathrm{mA}$. The oscillation wavelength is $-1.3 \mu \mathrm{m}$. The main feature of the light versus current characteristics ( $L-I$ curves) of the BC-DCC laser is the high nonlinearity. Fig. 2 shows experimental $\mathrm{CW} L-I$ curves of a typical BCDCC laser and a single-active-layer BC laser. The $L-I$ curve of the conventional $\mathrm{BC}$ laser is highly linear while that of the BC-DCC laser displays an optical switching behavior. An optical power jump of $2-3 \mathrm{~mW}$ at the threshold for BC-DCC lasers was observed. The external differential quantum efficiency in this region is much higher than that of the single-active-layer $\mathrm{BC}$ laser, and it can exceed $100 \%$ per facet. After the initial switch, there follows a region in which the $L-I$ curve of the BC-DCC 


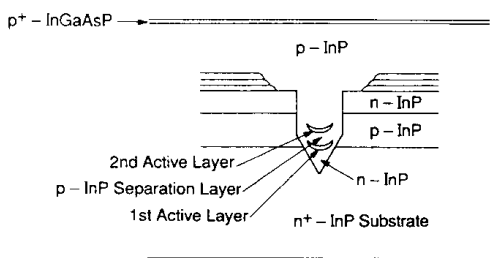

(a)

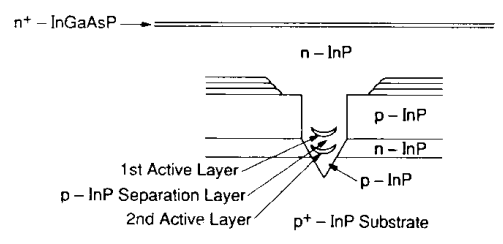

(b)
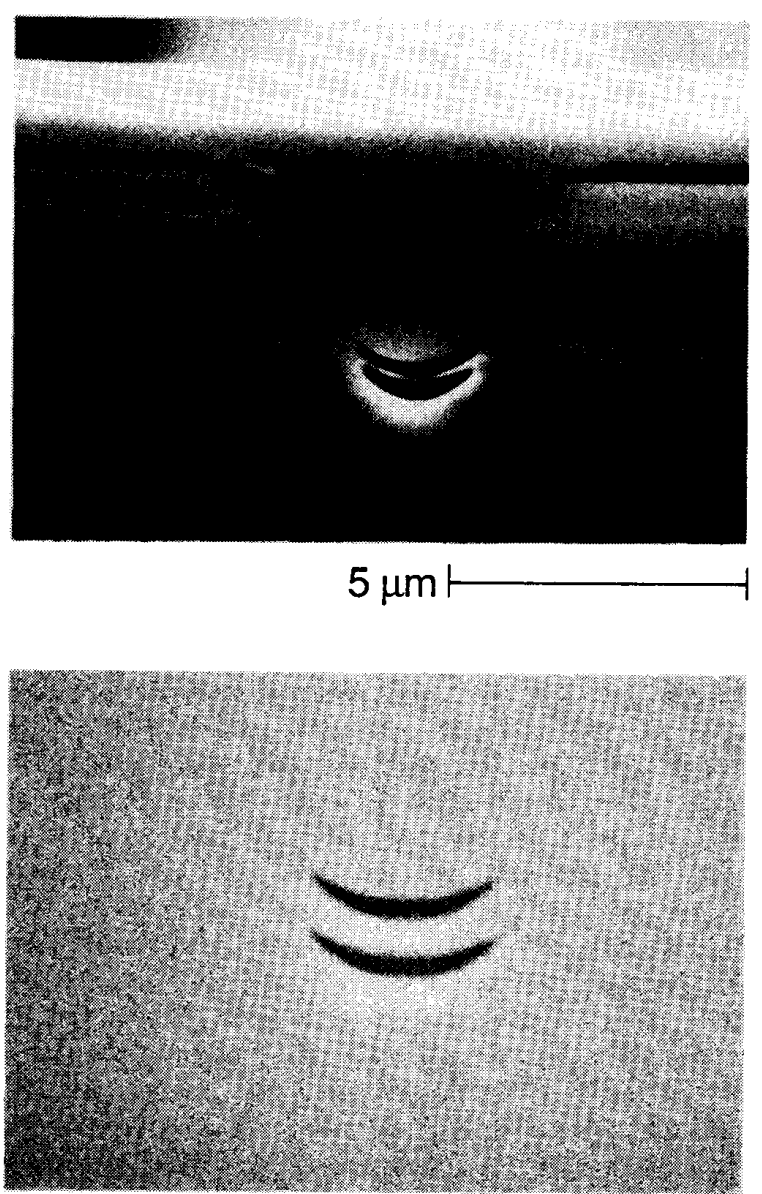

$2 \mu \mathrm{m}$

(c)

Fig. 1. Schematic structure of the BC-DCC laser. (a) BC-DCC laser on an $\mathrm{n}^{+}$-InP substrate. (b) BC-DCC laser on a $\mathrm{p}^{+}$-Inp substrate. (c) SEM pictures of the double-active layer.

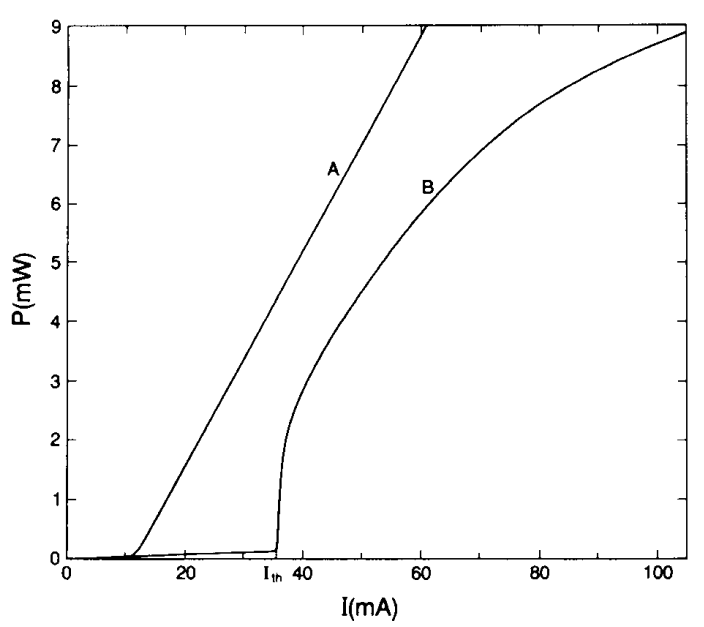

Fig. 2. $L-I$ characteristics of a $\mathrm{BC}-\mathrm{DCC}$ laser and a conventional $\mathrm{BC}$ laser.

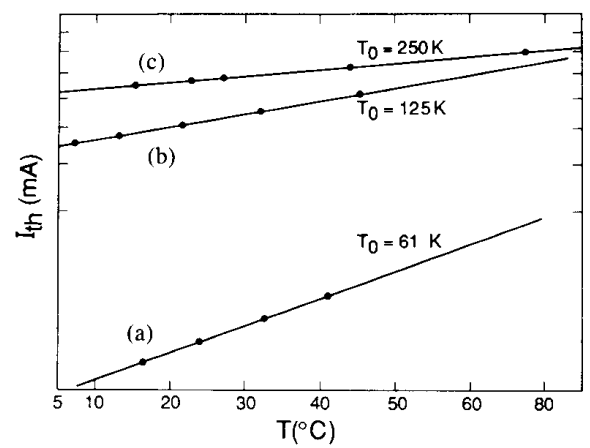

Fig. 3. $I_{\text {th }}$ as a function of the temperature for a conventional (a) $\mathrm{BC}$ laser on a $\mathrm{p}^{+}-\ln \mathrm{P}$ substrate showing $T_{0}=61 \mathrm{~K}$. (b) BC-DCC laser on a $\mathrm{p}^{+}$ InP substrate, showing $T_{0}=125 \mathrm{~K}$. (c) $\mathrm{BC}$-DCC laser on a ${ }^{+}-\mathrm{InP}$ substrate, showing $T_{0}=250 \mathrm{~K}$.

laser has a comparable slope as that of the single-activelayer BC laser. At higher current, the optical power gradually saturates. The power of the BC-DCC laser saturates much faster than the conventional $\mathrm{BC}$ laser.

It was noticed that the optical switching behavior of the BC-DCC laser is temperature dependent. At reduced temperature $\left(0^{\circ} \mathrm{C}\right.$ e.g. $)$, the switching is more pronounced. It gradually diminishes and almost disappears for a temperature above $\sim 50^{\circ} \mathrm{C}$.

Fig. 3 shows the temperature dependence of the threshold current of the BC-DCC laser and that of a conventional BC laser. For BC-DCC lasers on a p-InP substrate the characteristic temperature $T_{0}$ (assuming $I_{\mathrm{th}} \propto e^{T / T_{0}}$ ) ranges from 120 to $250 \mathrm{~K}$, depending on the laser parameters (the thickness of the active layers and the separation between the two active layers, e.g.), while the $T_{0}$ for $\sin$ gle-active-layer BC laser made from the same solution is typically $60 \mathrm{~K}$. The measured $T_{0}$ value for our BC-DCC lasers on an n-InP substrate is somewhat lower but still $\sim 30 \mathrm{~K}$ higher than that of the single-active-layer laser made on the same wafer. The possible reason for this is 
the junction shift due to the heating during the second growth cycle [5]. Also the separation between the two active layers for the wafers we processed seems too small [see Fig. 1(c), the upper picture]. It is obvious that there is an optimal separation between the two active layers for obtaining a high $T_{0}$ value. When the separation is very small or very big, the $T_{0}$ will approach that of a singleactive-layer laser.

\section{ANALYSIS}

To better understand the experimental results, we carried out an analysis using rate equations. Fig. 4 is a schematic picture of the band diagram for the device under high excitation. The equations which govern the carrier density $N_{i}$ in the $i^{\text {th }}$ active layer and photon density $\rho$ in the lasing mode are

$$
\begin{aligned}
\frac{d N_{1}}{d t}= & \frac{J_{1}}{e d_{1}}-B\left(N_{1}+N_{A 1}\right) N_{1}-A\left(N_{1}-N_{t r}\right) \rho \\
& -R_{\text {Auger, } 1} \\
\frac{d N_{2}}{d t}= & \frac{J_{2}}{e d_{2}}-B\left(N_{2}+N_{A 2}\right) N_{2}-A\left(N_{2}-N_{t r}\right) \rho \\
& -R_{\text {Auger }, 2} \\
\frac{d \rho}{d t}= & A\left[\Gamma_{1}\left(N_{1}-N_{t r}\right)+\Gamma_{2}\left(N_{2}-N_{t r}\right)\right] \rho-\frac{\rho}{\tau_{p}} \\
& +\beta B\left[\Gamma_{1} N_{1}\left(N_{1}+N_{A 1}\right)+\Gamma_{2} N_{2}\left(N_{2}+N_{A 2}\right)\right]
\end{aligned}
$$

where $R_{\text {Auger }, i}=C\left[\left(N_{i}+N_{A i}\right)^{2} N_{i}+\left(N_{i}+N_{A i}\right) N_{i}^{2}\right]$ [7] is the Auger nonradiative recombination rate, $J_{i}$ is the carrier recombination current in the $i^{\text {th }}$ active layer, $B$ is the radiative recombination constant, $N_{A i}$ is the acceptor density in the $i^{\text {th }}$ active layer, $A$ is the gain constant, $N_{\mathrm{tr}}$ is the transparency carrier density, $d_{i}$ is the $i^{\text {th }}$ active layer thickness, $\Gamma_{i}$ is the optical mode confinement factor of the $i^{\text {th }}$ active layer, $\tau_{p}$ is the photon lifetime, and $\beta$ is the coupling constant for spontaneous emission into the lasing mode.

The fourth equation needed for solving the problem is obtained by noticing that electrons escaping from the first active layer can either recombine in the second active layer or leak over the second active layer heterobarrier. Thus, the fourth equation is

$$
J_{\text {Leak, } 1}=J_{2}+J_{\text {Leak, } 2} \text {. }
$$

We model the leakage current similarly as in [7], where both the drift and the diffusion components of the leakage current are taken into account. According to [7], the electron leakage current can be expressed as

$$
J_{\text {Leak }}=\frac{\mu_{N}}{\mu_{P}} \frac{J_{P}}{N_{A l}} N_{l}-e D_{N}\left[\frac{A_{1}}{y_{1}}-\frac{A_{2}}{y_{2}}\right]
$$

where $\mu_{N}$ and $\mu_{P}$ are the electron and hole mobilities, $J_{P}$ is the hole current across the heterojunction, $N_{A 1}$ is the

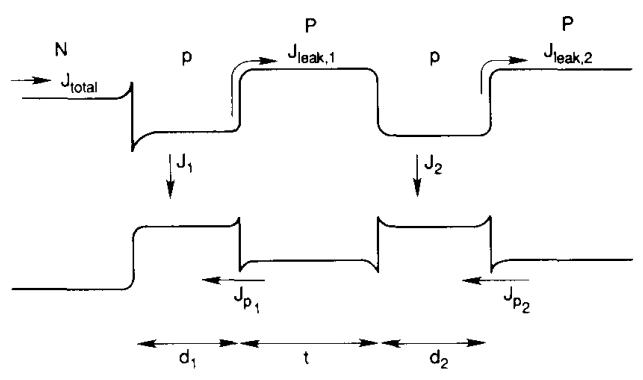

Fig. 4. The band diagram of a BC-DCC laser under high excitation.

ionized acceptor density, $D_{N}$ is the diffusion constant for electrons, and $A_{1}, A_{2}, y_{1}$, and $y_{2}$ are defined in [7]. It is important to note here that the leakage current is proportional to both the carrier concentration in the active layer and the hole current across the heterobarrier. Thus, the leakage keeps increasing above threshold even though the carrier concentration in the active region is clamped at its threshold value. Auger recombination produces hot electrons and we model these by using the electron temperature $T_{e}$ which is higher than the lattice temperature $T[8]$.

According to measurements [8], the electron temperature depends on the carrier concentration in the active layer, but the dependence starts to level off below $\sim 3$. $10^{18} \mathrm{~cm}^{-3}$. Because this is about the maximum carrier concentration in the present DCC lasers, we assume the same temperature for the carriers in both active layers. The electron temperature in the second active layer may be slightly lower than in the first active layer, but this causes only a slight decrease in the leakage current from the second active layer. As is explained later, this leakage current does not appreciably affect the temperature behavior of the DCC laser.

At higher temperatures, the Auger coefficient is larger so that the rate of generation of hot electrons by Auger recombination increases. Thus, the electron temperature must also increase. Lacking experimental data, we assume that since the Auger coefficient increases by $\sim 15 \%$ [11] in going from 300 to $330 \mathrm{~K}$, the electron temperature also increases by $-15 \%$ over the lattice temperature [7], i.e., it increases from $T_{e} \sim 335 \mathrm{~K}$ up to $T_{e} \sim 380 \mathrm{~K}$.

Solving (1)-(4) numerically with reasonable parameter values found in the literature, we obtained the carrier concentration $N_{1}$ and $N_{2}$, leakage currents $J_{\text {Leak. I }}$ and $J_{\text {Leak. 2 }}$, and the optical power $P$ (proportional to photon density $\rho$ ) as a function of the total injection current density $J_{\text {tot }}$ $=J_{1}+J_{\text {Leak, } 1}=J_{1}+J_{2}+J_{\text {Leak.2. }}$. It has been found that the functional behavior of $N, J_{\text {Leak }}$, and $P$ versus $J_{\text {tot }}$ depended strongly on the temperature $T$ and spontaneous emission factor $\beta$. The calculated results are summarized in Figs. 5 to 7.

Shown in Fig. 5(c) is the optical power as a function of the total injection current for room temperature operation. The value of $\beta$ used for this calculation is $10^{-4}$ which is supposed to be typical for a real index-guided DH laser like our BH laser [9]. A sharp rise in the optical power at 


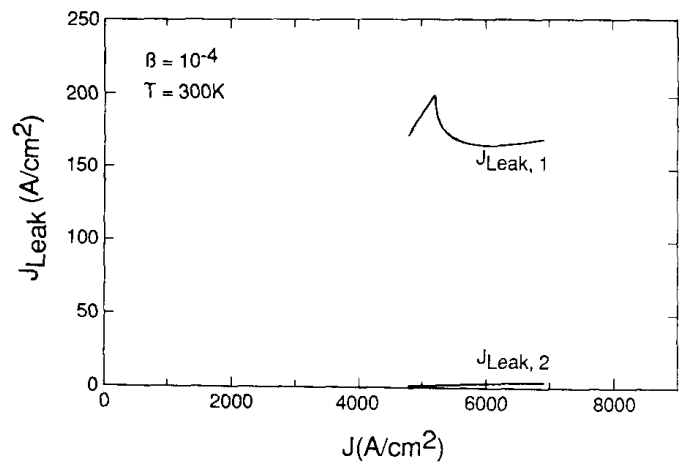

(a)

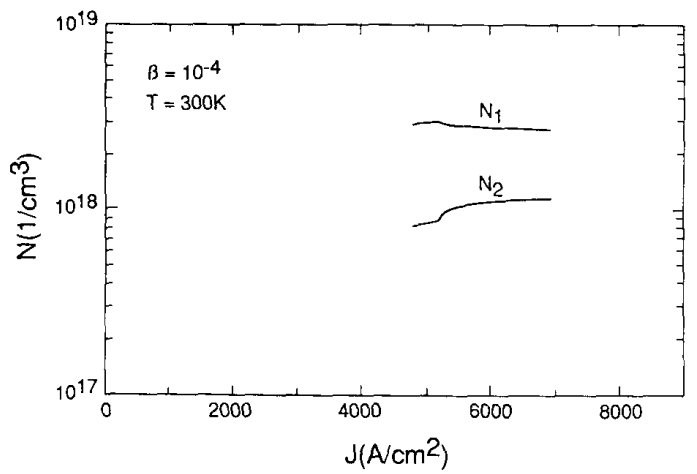

(b)

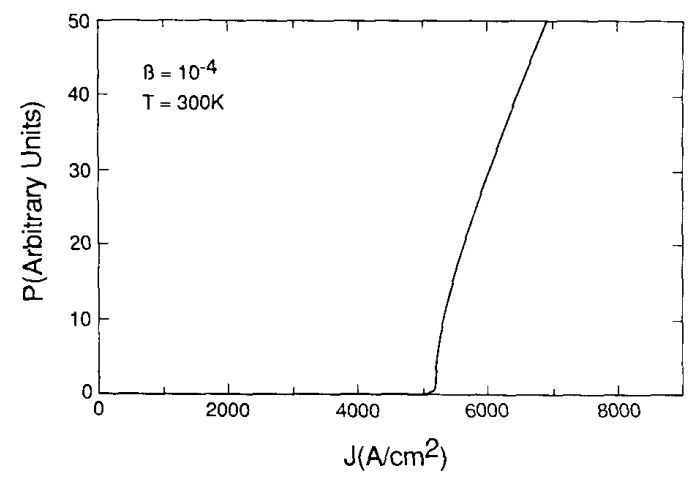

(c)

Fig. 5. Calculated: (a) Leakage current ( $J$ Leak). (b) Carrier concentration in the active layers $\left(N_{1}, N_{2}\right)$. (c) Optical power (p) as a function of total injection current $(J)$, assuming $\beta=\omega^{-4}, T=300 \mathrm{~K}$.

threshold is evident. At elevated temperature (e.g., 330 $\mathrm{K}$ ), in addition to the threshold current increase, the output power jump at threshold diminishes and disappears as can be seen from Fig. 6(c). These all agree very well with our experimental observation.

In gain-guided lasers, the spontaneous emission parameter $\beta$ is much larger. Assuming $\beta=5 \cdot 10^{-3}$, we calculated optical power as a function of injection current. The result is plotted in Fig. 7(c). The sudden jump of the output power at threshold disappears consistent with earlier experimental results [5] with gain-guided lasers.

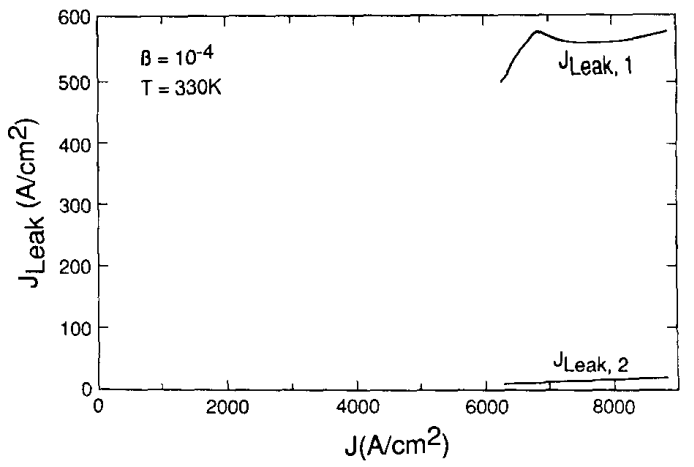

(a)

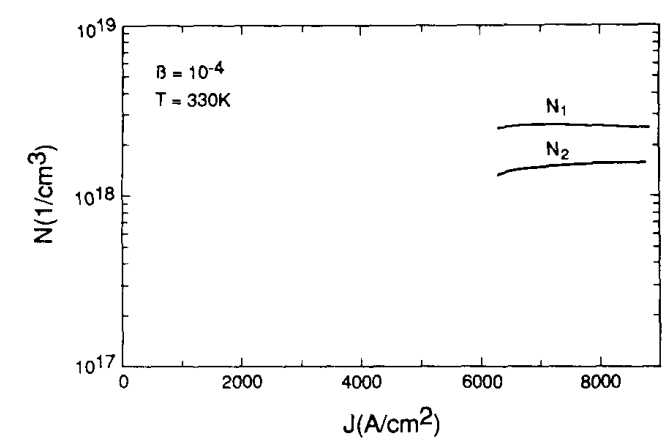

(b)

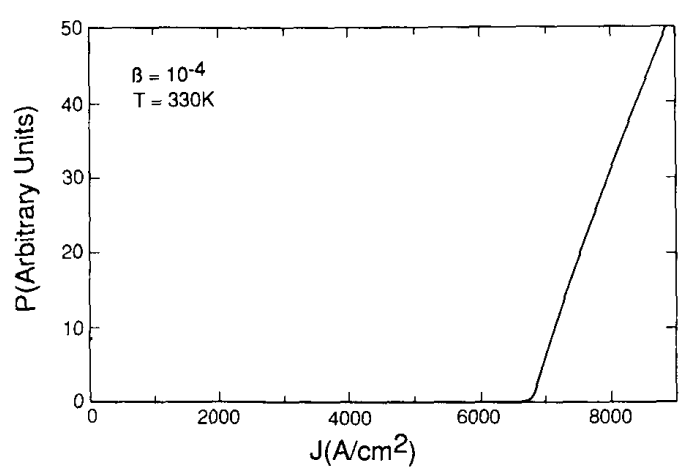

(c)

Fig. 6. Calculated: (a) Leakage current ( $J$ Leak). (b) Carrier concentration in the active layers $\left(N_{1}, N_{2}\right)$. (c) Optical power $(P)$ as a function of total injection current $(J)$, assuming $\beta=10^{-4}, T=330 \mathrm{~K}$.

\section{Discussion}

The optical switching behavior of the BC-DCC laser can be qualitatively understood. The second active layer which is placed closely to the first active layer serves as a saturable absorber for the lasing mode. When DCC laser approaches its threshold, the photon density increases. The second active layer is bleached to near transparency. This accounts for the optical power jump at threshold. In fact, below threshold $J_{\text {Leak, 1 }}<<J_{1}, J_{2}=J_{\text {Leak, 1 }}-J_{\text {Leak, 2 }}$ $\ll J_{1}$, therefore $N_{2} \ll N_{1}$, and also $N_{2} \ll N_{\text {tr }}$. The net effect of the second active layer is absorbing as can be 


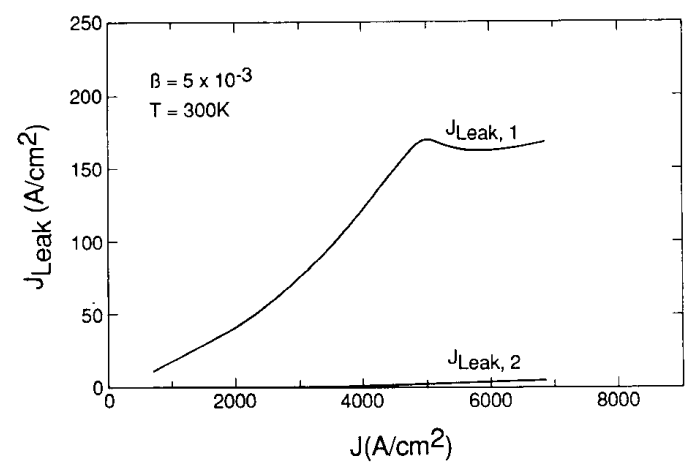

(a)

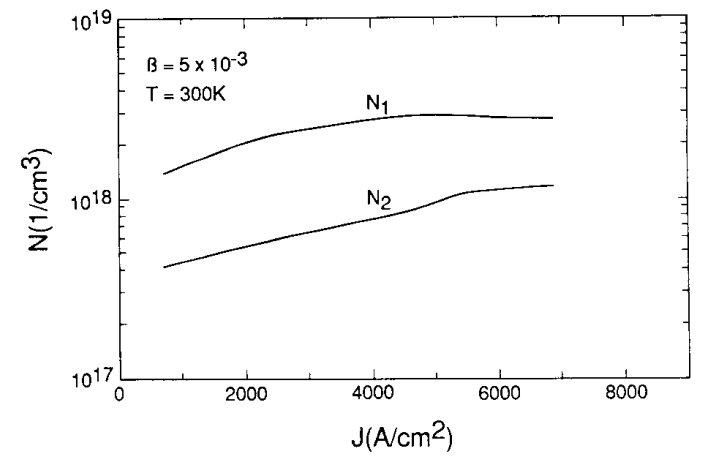

(b)

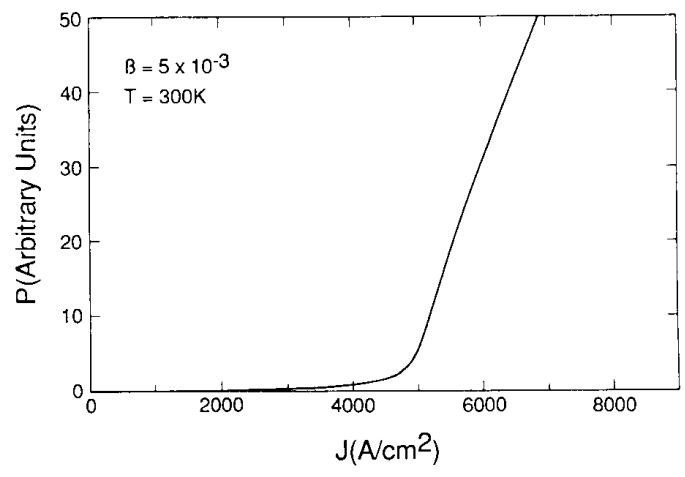

(c)

Fig. 7. Calculated: (a) Leakage current ( $J$ Leak). (b) Carrier concentration in the active layers $\left(N_{1}, N_{2}\right)$. (c) Optical power $(P)$ as a function of total injection current $(J)$, assuming $\beta=5 \cdot 10^{-3}, T=300 \mathrm{~K}$.

seen from the second term in (3)

$$
\frac{d \rho}{d t}=\cdots+\Gamma_{2} A\left(N_{2}-N_{\mathrm{tr}}\right) \rho+\cdots .
$$

At (or slightly above) threshold, the photon density $\rho$ increases, this causes the absorption term to become larger. The result is the carrier density in the second active layer, $\mathrm{N}_{2}$, increases rapidly [see the third term of (2)]. This reduces the optical loss due to the second active layer thus requiring a smaller gain from the first layer which causes $N_{1}$ to decrease. $N_{1}+N_{2}$ will be clamped above threshold. In Fig. 5(b), a step increase in $N_{2}$ and a step decrease in
$N_{1}$ can be seen clearly. Showing in Fig. 5(a) is the leakage current versus total injection current. The leakage current from the first active layer has a step decrease at threshold. At higher temperature, the leakage current from the first active layer increases and this increases $N_{2}$. Thus the second active layer becomes less absorbing even below threshold. This in turn diminishes the step increase in $\mathrm{N}_{2}$ [see Fig. 6(b)], as well as the jump in the output power [see Fig. 6(c)].

With a higher value of $\beta$, the photon density $\rho$ starts to increase well below threshold because of larger spontaneous emission into the lasing mode. This causes $N_{2}$ to increase and become much larger below threshold than it is for small $\beta$. The step increase in $N_{2}$ and an output power jump at threshold is now washed out as can be seen from Fig. 7(b) and 7(c).

The $T_{0}$ value for BC-DCC laser can be as high as 180 $250 \mathrm{~K}$ as shown by our measurements as well as by previous experiments [4]-[5]. This anomalously high $T_{0}$ can not be attributed solely to the elimination of the carrier leakage in the present DCC structure. As a matter of fact, a "leakage free"' DH InGaAsP-InP laser will have a $T_{0}$ value only up to $90-100 \mathrm{~K} \mathrm{[7],} \mathrm{[10].} \mathrm{The} \mathrm{important} \mathrm{factor}$ is that Auger recombination accounts for about half of the total injected current. It is the suppression of nonradiative Auger process in this particular configuration that produces a very high $T_{0}$.

In this work, the Auger process is described as $J_{\text {Auger }} \propto$ $\mathrm{CN}^{3}$, where $C$ is the Auger constant and $N$ is the carrier concentration. Both depend on temperature [11]. For a conventional DH laser such as a single-active-layer BC laser, at the temperature $T$, the Auger recombination at or above threshold can be written as $J_{\text {Auger }} \propto \mathrm{CN}^{3}$, where $N$ is the threshold carrier concentration. At $T^{\prime}>T, J_{\text {Auger }}$ $\propto C^{\prime} N^{\prime 3}$, where $C^{\prime}>C$ and $N^{\prime}>N$. For DCC laser at the temperature $T, J_{\text {Auger }} \propto C\left(N_{1}^{3},+N_{2}^{3}\right)$, where $N_{1}+$ $N_{2}=2 N_{0}$, where $N_{0}$ is the "average"' threshold carrier density. At the temperature $T^{\prime}>T, J_{\text {Auger }} \propto C^{\prime}\left(N_{1}^{\prime 3}+\right.$ $N_{2}^{\prime 3}$ ). However, our calculation showed that carrier leakage from the first active layer to the second active layer increases so fast with temperature that at elevated temperature $N_{1}^{\prime}$ does not differ too much from $N_{1}$. Thus $N_{2}^{\prime}$ acquires almost all the increase in the total carrier concentration to obtain the desired gain, i.e., $N_{2}^{\prime} \approx 2 N_{0}^{\prime}-$ $N_{1}$, where $2 N_{0}^{\prime}=N_{1}^{\prime}+N_{2}^{\prime}, N_{0}^{\prime}$ is the average threshold carrier density at $T^{\prime}$. As $N_{2}$ is generally much smaller than $N_{\text {I }}$ or $N_{0}$, the total effect is that $J_{\text {Auger }}$ for DCC laser increases with increasing temperature much less than $J_{\text {Auger }}$ for a conventional DH laser. This can be seen by comparing Figs. 5(b) and 6(b) and is also illustrated clearly in the following calculated numerical example. At room temperature $(300 \mathrm{~K})$, for our BC-DCC laser the threshold carrier concentrations $N_{1}$ and $N_{2}$ are $3.0 \cdot 10^{18} \mathrm{~cm}^{-3}$ and $0.89 \cdot 10^{18} \mathrm{~cm}^{-3}$, respectively. At the temperature $T^{\prime}=$ $330 \mathrm{~K}, N_{1}^{\prime}$ and $N_{2}^{\prime}$ become $2.61 \cdot 10^{18} \mathrm{~cm}^{-3}$ and $1.67 \cdot$ $10^{18} \mathrm{~cm}^{-3}$, respectively. We can see that $N_{1}^{3}+N_{2}^{3} \propto 27.7$ and $N_{1}^{3}+N_{2}^{\prime 3} \propto 22.6$. On the other hand, for a singleactive-layer BC laser, at $T=300 \mathrm{~K}, N=1.86 \cdot 10^{18}$ 
$\mathrm{cm}^{-3}$, and at $T^{\prime}=330 \mathrm{~K}, N^{\prime}=2.06 \cdot 10^{18} \mathrm{~cm}^{-3}$. Then we have $N^{3} \propto 6.43$, and $N^{\prime 3} \propto 8.74$. The relative increase for $N^{3}$ for a single-active-layer BC laser is $36 \%$, while for a BC-DCC laser $N_{1}^{3}+N_{2}^{3}$ even decreases by $18 \%$ with increasing temperature. Combining with the increase in Auger constant $C$ ( $30 \%$ from 300 to $330 \mathrm{~K}$ ), this accounts for the temperature behavior of the $\mathrm{BC}$ and BC-DCC lasers. Indeed, the calculated $T_{0}$ for our BCDCC laser is $175 \mathrm{~K}$ which falls into the range of experimental data, whereas calculated $T_{0}$ for an ordinary BC laser is about $80 \mathrm{~K}$. For spontaneous recombination part of the total current, the same argument applies.

As for the increased threshold current of BC-DCC laser compared to a conventional $\mathrm{BC}$ laser, the introduction of a second-active-layer causes, in addition to increased optical loss, the optical confinement factor of the first active layer to become smaller than for a single-active-layer laser having similar active layer. This necessitates a bigger density as well as a bigger number of carriers in the firstactive layer thus leading to the increase in threshold current.

\section{Conclunsion}

The initial idea for DCC structure is to construct a second active layer to collect the leaked carriers from the first-active layer and reuse them for radiative recombination. Our calculations showed that for reasonable laser operation parameters, at threshold the second active layer was bleached to near transparency but never contributes to gain. This accounts for the optical switching behavior for BC-DCC laser at room temperature. The way the second-active layer contributes to the improved $T_{0}$ is twofold. It does collect the leaking carriers, thus becoming less lossy at higher temperature and partially counteracting the threshold increase due to higher Auger rates at increased temperature. Also, the presence of the secondactive layer offers a possibility that at elevated temperature the carriers redistribute themselves among the two active layers in such a way that the increase in Auger recombination is much suppressed. This accounts for the anomalously high $T_{0}$ value measured for DCC structure.

\section{ACKNOWLEDGMENT}

The authors acknowledge D. Armstrong for his technical help in the $T_{0}$ measurement.

\section{REFERENCES}

[1] I. Mito, M. Kitamura, K. Kobayashi, K. Kobayashi, and R. Lang "High $T_{0}$ and high temperature CW operation in InGaAsP DC-PBH LD," 8th Int. Semicond. Laser Conf., Paper 28, Ottawa, Canada, 1982.

[2] T. R. Chen, L. C. Chiu, K. L. Yu, U. Koren, A. Hasson, S. Margalit, and A. Yariv, "Dependence of $T_{0}$ on $P$-cladding layer doping level in InGaAsP lasers," Japan. J. Appl. Phys., vol. 22, p. L559, 1983.

[3] E. A. Rezek, N. Holonyak, and B. K. Fuller, "Temperature dependence of threshold current for coupled multiple quantum-well In GaAsP-InP heterostructure laser diodes," Japan. J. Appl. Phys., vol. 22, p. L559, 1983.

[4] M. Yano, Y. Nishitani, K-I. Hori, and M. Takusagawa, "Temperature characteristics of double-carrier-confinement (DCC) heterojunc- tion InGaAsP $(\lambda=1.3 \mathrm{~mm})$-InP lasers," IEEE J. Quantum Electron., vol. QE-19, p. 1319, 1983.

[5] T. C. Hasenberg and E. M. Garmire "Characteristics of InGaAsPInP $p$-DCC lasers grown by the three-melt technique," IEEE $J$. Quantum Electron, vol. QE-23, p. 948, 1987.

[6] E. Oomura, H. Higuchi, Y. Sakakibara, R. Hirano, H. Namizaki, W. Susaki, K. Ikeda, and K. Fujikura, "InGaAsP-InP buried crescent laser diode emitting at $1.3 \mu \mathrm{m}$ wavelength," IEEE J. Quantum Electron., vol. QE-20, p. 866, 1984.

[7] L. C. Chiu, K. L. Yu, S. Margalit, T. R. Chen, U. Koren, A. Hasson, and A. Yariv, "Field and hot carrier enhanced leakage in InGaAsP-InP heterojunctions," IEEE J. Quantum Electron., vol. QE-19, p. 1335, 1983.

[8] O. Wada, S. Yamakoshi, and T. Sakurai, "Band-gap enhanced carrier heating in InGaAsP/InP double heterostructure light-emitting diodes," Appl. Phys. Lett., vol. 41, p. 981, 1982.

[9] C. Harder, K. Y. Lau, and A. Yariv, "Bistability and pulsations in semiconductor lasers with inhomogeneous current injection," IEEE J. Quantum Electron., vol. QE-18, p. 1351, 1982.

[10] H. C. Casey, Jr., "Temperature dependence of the threshold current density in InP- $\mathrm{Ga}_{0.28} \mathrm{In}_{0.72} \mathrm{As}_{0.6} \mathrm{P}_{0.4}(\lambda=1.3 \mu \mathrm{m})$ double heterostructure lasers," J. Appl. Phys. vol. 56, p. 1959, 1984.

[11] A. Haug, "Evidence of the importance of Auger recombination for InGaAsP lasers," Electron. Lett, vol. 20, p. 85, 1984.

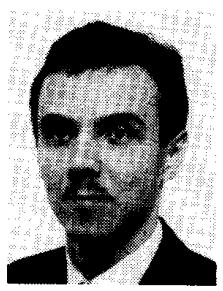

Markus Kajanto was born in Helsinki, Finland in 1961. He received the M.Sc. degree and the Licentiate degree in technical physics from the Helsinki University of Technology, Espoo, Finland, in 1985 and 1986, respectively. From 1986 to 1989 he was a graduate student at the California Institute of Technology, Pasadena, where he studied and conducted research on III-V semiconductor lasers. He received the Ph.D. degree in 1988 .

He is currently working as a management consultant with McKinsey \& Company, Inc., Stockholm, Sweden, and is also engaged in M.B.A. studies at I.N.S.E.A.D., Fontainebleau, France.

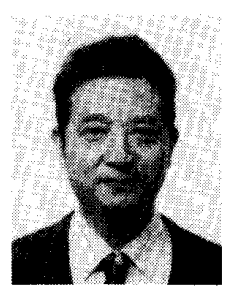

T. R. Chen was born in China on December 4 1939. He graduated from Wuhan University in 1962 with a degree in physics.

He then joined the faculty of the University of Electronic Science and Technology in China, becoming a full professor in 1984 . He was appointed a visiting professor at the University of Illinois, Urbana-Champaign, for the summer of 1981 , and was appointed a visiting associate in the Department of Applied Physics, California Institute of Technology, Pasadena, from 1980 to 1983 and again from 1985 until the present. His research interests have been in the area of optical resonator theory, optical communication, semiconductor photonic devices, and optoelectronic integration. He is an author or coauthor of three books (in Chinese) and approximately 60 professional papers.

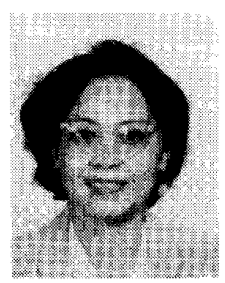

Y. H. Zhuang was born in China on December 15,1939 . She graduated from the Chengdu University of Science and Technology in 1962 with a degree in engineering mechanics.

She then joined the faculty of the University of Electronic Science and Technology in China. She is presently a Research Engineer with the Department of Applied Physics, California Institute of Technology, Pasadena. Her current research interets are in the area of semiconductor photonic devices.

Amnon Yariv (S'56-M'59-F'70), for a photograph and biography see $\mathrm{p}$ 1190 of the July 1990 issue of this Journal. 